\title{
Assemble, extrude and reform
}

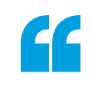

The print
resolution

achieved

using our new

method is

competitive

3D printers with high-end

3D printing can create an impressive range of intricate $3 \mathrm{D}$ architectures; however, current 3D printing technologies are, in general, not compatible with functional small molecules and hence this function and any supramolecular arrangements are not well preserved after $3 \mathrm{D}$ printing. If smart objects and devices are to be 3D printed from smart materials with, for example, host-guest interactions, selfassembled molecular components or electron-transporting capabilities, new approaches are required.

Now, Chenfeng Ke and colleagues report the $3 \mathrm{D}$ printing of viscoelastic hydrogels comprising assembled superstructures of molecular monomers and a polymeric template. "As expected, the extrusion process

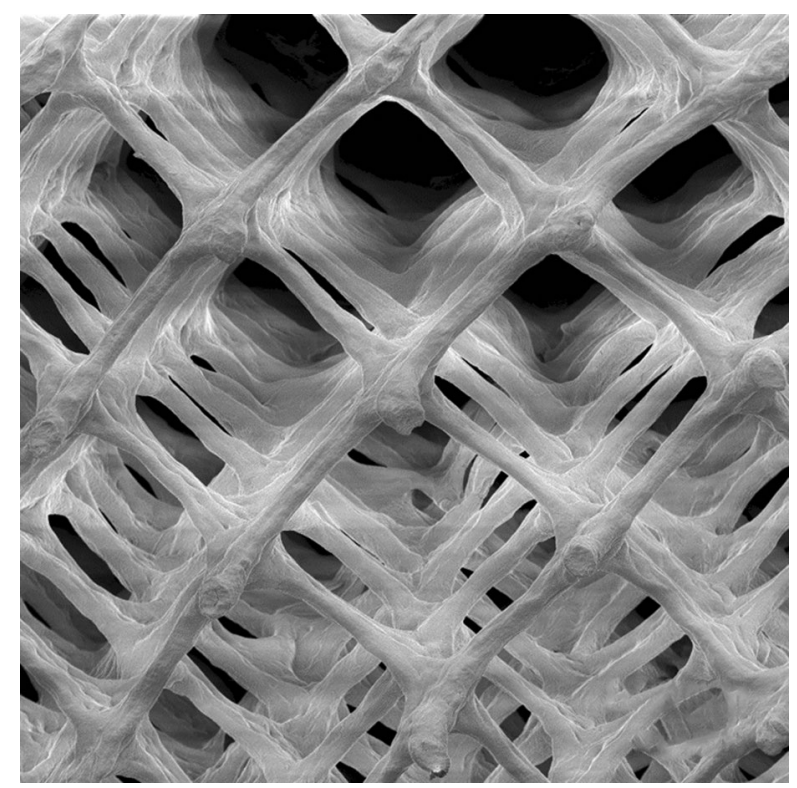

Reproduced with permission from Li, L. et al. (2018), John Wiley and Sons disrupts the assemblies; however, they are reformed in the subsequent step," says Ke. The approach involves the direct ink writing of a hydrogel ink comprising monomer-template conjugates, followed by evaporationinduced assembly of the conjugates. Then, the assembled system is either thermally or photocrosslinked, depending on the type of monomer used in the fabrication. Finally, the polymer template is removed by calcination or extraction to give homogeneous 3D-printed monoliths with molecular-scale function.

The range of monomers and polymeric templates suitable for this approach is likely to be considerable. So far, the researchers use Pluronic as the polymer template, and either inorganic monomers (tetraethyl orthosilicate) or organic monomers (benzene-tricarboximide derivative) in the inks. "For this printing approach to be successful, there is a narrow viscoelastic window for the monomer-template hydrogel," explains Ke. "The viscoelasticity must permit extrusion and enable the hydrogel to recover quickly and be sufficiently strong to support itself, as well as to allow the assembly of the supramolecular arrangement during evaporation." As a result, for individual monomers with different chemical properties, this viscoelastic window has to be identified in each case.

"The print resolution achieved using our new method is competitive with high-end 3D printers," says Ke. "More specifically, the printing resolution is about $30 \mu \mathrm{m}$ after the evaporation and crosslinking stages, which is about a 10 times higher resolution compared with the just printed gel," says Ke. "The gel has the ability to shrink uniformly as a consequence of the introduction of a few sacrificial layers."

By doping the hydrogel inks with fluorescent tags, Ke and colleagues establish the details of the mechanism of the co-assembly process. The interactions between the template and monomers are relatively strong and hence the co-assembly process is localized. "The tagged monomer molecules can be seen to rapidly assemble at the surface of the hydrogel and then increasingly start to reorganize close to the inner sections of the printed ink," says Ke.

In the future, Ke and colleagues envisage the introduction of biological macromolecules, such as DNA or peptides, into the starting materials, with the aim of fabricating 3D printed architectures in which the function and the hierarchical structure arrangement of these biological molecules are retained following the printing step. "Also, if we can integrate two or more functional molecules into one gel or spatially separate the printing of two different gels within one object, our approach could enable multimaterial printing to produce multiresponsive smart devices," concludes Ke.

Alison Stoddart

ORIGINAL ARTICLE Li, L. et al. Hierarchical co-assembly enhanced direct ink writing. Angew. Chem. Int. Ed. https://doi.org/10.1002/ anie.201800593 (2018) 